\title{
EPIDEMIOLOGIA DELL'INFEZIONE DA TOXOPLASMA IN DONNE ITALIANE E STRANIERE NEL PERIODO 2000-2004
}

Clerici P., De Paschale M., Cavallari S., Mariani G., Agrappi C., Mirri P.,Viganò E.F.

U.O. Microbiologia, Ospedale Civile di Legnano,

Via Candiani 2, 20025 Legnano (MI)

Introduzione. La prevalenza di $\mathrm{Ab}$ anti Toxoplasma varia nel mondo: è minore nei paesi industrializzati del Nord Europa ed è maggiore nei paesi in via di sviluppo. In Italia si è osservata una progressiva diminuzione della prevalenza, per classi di età, dagli anni '80 al 2000.

Scopo del lavoro è lo studio della prevalenza di $\mathrm{Ab}$ anti Toxoplasma in donne fertili italiane e straniere afferenti a Legnano nel periodo 2000-2004.

Metodi. È stata effettuata la ricerca di Ab anti Toxoplasma (ETI-TOXO G PLUS, Dia Sorin) in 8167 donne tra i 20 e i 39 anni (un solo esito per paziente) sottoposte a screening sierologico (per gravidanza), di cui 814 straniere (10\%).

Risultati. La prevalenza di IgG anti Toxoplasma nelle 8167 donne è stata del $25.7 \%$ ed è passata dal $30,5 \%$ nel 2000 al $22,9 \%$ nel $2004(p<0,01)$. Le donne straniere costituivano il $6,9 \%$ del campione nel 2000 e sono arrivate al $12,7 \%$ nel $2004(p<0,01)$. La sieroprevalenza nelle donne italiane è passata dal 30,6\% nel 2000 al 20,9\% nel $2004(p<0,01)$, mentre nelle straniere la sieroprevalenza è stabile attorno al $37 \%$. Nelle italiane si osserva un progressivo aumento della prevalenza passando dalla classe 20-24 anni (20,2 \%) alla classe 35-39 anni (32\%, p<0,01); per le straniere si passa dal 32.3 $\%$ al $38 \%$ (NS) nella classe 35-39 anni.

Sempre nelle italiane dal 2000 al 2004 si osserva una significativa diminuzione $(\mathrm{p}<0,01)$ nelle positività, mentre non si osservano trend nelle straniere.

Conclusioni. Si conferma la diminuzione, dal 2000 al 2004, di immunità alla Toxoplasmosi nelle donne, ma si evidenziano nette differenze tra italiane e straniere nella prevalenza e nel trend. 